\title{
Comparison of formulation costs for poultry feeds based on crude protein versus the digestible lysine to protein ratio
}

\section{Comparação dos custos de formulação para aves baseados na proteína bruta versus relação lisina digestível proteína}

\author{
Jomara Broch ${ }^{*}$ (D) , Ricardo Vianna Nunes ${ }^{1}$ (D) , Rashed Abdullah Alhotan² (D) , Gene \\ Michael Pesti ${ }^{3}$
}

${ }^{1}$ Universidade Estadual do Oeste do Paraná, Marechal Cândido Rondon, PR, Brazil

${ }^{2}$ King Saud University, Riade, Ar Riyad, Saudi Arabia

${ }^{3}$ Georgia University, Athens, Georgia, EUA

${ }^{*}$ Correspondent - brochjomara@yahoo.com.br

Section: Zootecnia

Received

June 3, 2019

Accepted

August 7. 2019.

Published

August 6, 2020

www.revistas.ufg.br/vet visit the website to get the how to cite in the article page.

\begin{abstract}
The objective of this study was to determine the influence of using dLys:TP instead of crude protein recommendations of commercial poultry breeding companies to meet the chicken's need for dietary essential and non-essential amino acids. The ingredient composition matrix used in the feed formulation was compiled from Feedstuffs Magazine. The rations were formulated using a linear feed formulation program to meet the minimum cost, with crude protein or dLys: TP of $5.58 \%$. Using the dLys to TP ratio versus CP minimums for the model diets chosen, formula costs were changed from $+\$ 10.96$ to -\$4.26 per ton of feed. The magnitude of the changes was dependent on the imbalance present in the feeds from using the breeder management guide recommendations and ingredient prices. Through the use of dLys: TP, it is possible to improve the feed formulation process, assuring the birds get adequate EAA and NEAA without being wasteful and reducing environmental pollution.
\end{abstract}

Key Words: amino acids; concentration; digestibility.

\begin{abstract}
Resumo
O objetivo deste estudo foi determinar a influência do uso de lisina digestível: proteína verdadeira (LISd: PV) em vez de recomendações de proteína bruta de empresas de criação de aves comerciais para atender à necessidade de aminoácidos essenciais e não essenciais. A matriz de composição de ingredientes utilizada na formulação de alimentação foi compilada a partir da Revista de Ingredientes. As rações foram formuladas utilizando um programa de formulação de ração linear para suprir o custo mínimo, com proteína bruta ou dLis: PV de $5,58 \%$. Usando a relação LISd para PV versus os mínimos PB para as dietas de modelo escolhidas, os custos de fórmula foram alterados de $+\$ 10,96$ para $-\$ 4,26$ por tonelada de alimento. A magnitude das mudanças depende do desequilíbrio presente nos ingredientes usando as recomendações do guia de manejo do reprodutor e os preços dos ingredientes. Por meio do uso de dLis: PV, é possível melhorar o processo de formulação de rações, assegurando
\end{abstract}


que as aves obtenham adequados níveis de aminoácidos essenciais (AAE) e aminoácidos não essenciais (AANE) sem desperdício e reduzindo a poluição ambiental.

Palavras-chave: Aminoácidos. Concentração. Digestibilidade.

\section{Introduction}

Dietary protein is considered one of the main components of the cost of commercial feeding of birds. According to Corzo et al. ${ }^{(1)}$ a reduction in dietary protein level and the use of supplemental purified amino acids make it possible to reduce this cost. However, it is important to take care that the composition of AA in diets can vary according to factors such as ingredient inclusion, different sources of protein, inclusion of synthetic $A A$, concentrations of these $A A$ and the ratio of $A A$ : Lys, as well as the expression of $A A$ in the total or digestible protein base(2).

Current formulation models and recommendations often include minimum restrictions for crude protein and amino acids or only amino acids ${ }^{(3,4)}$. The crude protein contents of ingredients have been estimated by multiplying the total nitrogen of the ingredient (determined by the Kjeldahl or Dumas methods) by an universal nitrogen to protein ( $\mathrm{N}$ : CP) conversion factor of $6.25^{(5)}$.

However, this factor (6.25) was validated for milk products, but nutritionists applied it to other ingredients without similar scrutiny. This practice introduces error, the $\mathrm{N}$ content of casein (milk protein) is quite different from all the other common feed ingredients fed to poultry and livestock. It is important to consider that the N: CP conversion factor of the ingredients may vary depending on the composition of amino acids, the nitrogen content of each amino acid, and the presence of other organic nitrogen compounds such as nucleic acids, urea, ammonia, phospholipids and nitrates etc., which also need to be accounted for ${ }^{(6)}$.

In the $21^{\text {st }}$ century, the amino compositions of thousands of feed ingredient samples have been determined so it is now possible to estimate the true protein (TP) and N: CP conversion factor for each ingredient. A problem with typical linear feed formulation models for poultry has been the inability to adequately represent the non-essential amino acids since the actual supply available to the bird is the sum of the non-essential amino acids present in the diet and the excess of the essential amino acids not incorporated into protein and therefore available to supply amino groups for the de novo synthesis of the non-essential amino acids.

Diets formulated with commercially available sources of Lys, Met and Thr allow a significant reduction in $\mathrm{CP}^{(2)}$. With this reduction, the AA-to-Lys ratios are improved. Thus, the costs of excretion for those AA not incorporated into protein and feed costs are eliminated. Therefore, the formulation of diets based on digestible AA is considered by many researchers as the best way of measuring the value of AA in the ingredients, as it allows for the reduction of CP and considerably reduces the cost of diets, besides reducing the excretion of pollutants in the excreta. 
From a meta-analysis of 70 datasets taken from broiler experiments compiled from the world's literature, Alhotan and Pesti( ${ }^{(7)}$ determined the ratio of dLys to TP for maximum growth and minimum feed conversion ratio to be $5.58 \%$. By using TP instead of CP and the dLys:TP ratio, the protein content of the ingredients is more accurately represented and the excess of essential AA available for the de novo synthesis of non-essential AA is represented as well.

The objective of these analyses was to evaluate the influence of using dLys:TP during feed formulation instead of crude protein recommendations of commercial poultry breeding companies to meet the chicken's need for dietary essential and non-essential amino acids, and the impact of the different formulations on feed costs.

\section{Materials and methods}

The ingredient composition matrix used in the feed formulations was compiled from the Feedstuffs Magazine ingredient analysis tables (Tables 1 and 2) ${ }^{(8)}$.

Diets for growing broilers were formulated with standardized illeal digestibility (SID)(9) values, and for mature birds with values from cecectomized roosters $(C R)^{(10)}$. The costs of the ingredients used were provided by a major American integrator during the Spring of 2017 (Table 1).

The least-cost feeds were formulated using Microsoft Excel and WUFFDA 2.0 (Windows User Friendly Feed Formulation)(11). Sensitivity analysis was implemented with the Solver add-in of Excel. The ratio of dLys to TP was fixed at 5.58\% (or TP / dLys $=17.92$ ) ${ }^{(7)}$. Two groups of diets were formulated; one group was based on the dLys:TP ratio and the other was based on CP minimums.

Experimental diets were formulated for 1) Ross 308 starting chickens (1-10 d), 2) Cobb 700 finishing broilers (43+ d), 3) Hy-Line W-36 starting pullets (0- 3 wks), 4) Ross 708 breeders 1,5) Hy-Line W-36 peaking layers and 6) Hy Line W-36 Layers $5(<80 \%)$, according to breeder management guides (Table 3). The rations were formulated in order to meet the nutrient specifications at minimum cost. The availability of the nutrients was the same for the various rations formulated, differing only in the requirements for crude protein or digestible lysine: true protein. 
Comparison of formulation costs for poultry feeds based on crude protein versus the digestible lysine to protein ratio Broch J. et al.

Table 1. Ingredient costs, restrictions and compositions (except protein and amino acids) used for the formulations (\%). Feeds for growing broilers were formulated with standardized illeal digestibility (SID) values and for mature birds with values from cecectomized roosters (CR)

\begin{tabular}{|c|c|c|c|c|c|c|c|c|c|c|c|}
\hline Ingredients & Cost, \$/ton & Min & Max & $\begin{array}{c}\text { Dry } \\
\text { Matter }\end{array}$ & $\begin{array}{c}\text { Met. } \\
\text { Energy }\end{array}$ & $\mathrm{Ca}$ & $\begin{array}{c}\text { Total } \\
\mathbf{P}\end{array}$ & $\begin{array}{c}\text { Avail. } \\
\text { P }\end{array}$ & $\mathrm{Na}$ & $\mathrm{Cl}$ & Choline \\
\hline Corn [SID] & 150 & 0 & 100 & 86.0 & 3.37 & 0.01 & 0.28 & 0.12 & 0.02 & 0.04 & 1.10 \\
\hline Corn $[\mathrm{CR}]$ & 150 & 0 & 100 & 86.0 & 3.37 & 0.01 & 0.23 & 0.09 & 0.02 & 0.04 & 1.10 \\
\hline Soybean meal ( $48 \%$ CP) [SID] & 346 & 0 & 100 & 88.0 & 2.46 & 0.31 & 0.72 & 0.24 & 0.04 & 0.02 & 2.85 \\
\hline Soybean meal (48\% CP) [CR] & 346 & 0 & 100 & 88.0 & 2.46 & 0.31 & 0.67 & 0.21 & 0.04 & 0.02 & 2.85 \\
\hline Corn DDGS [SID] & 124 & 0 & 5 & 90.2 & 2.85 & 0.03 & 0.77 & 0.54 & 0.19 & 0.17 & 3.40 \\
\hline Corn DDGS [CR] & 124 & 0 & 10 & 92.0 & 2.80 & 0.14 & 0.89 & 0.55 & 0.20 & 0.17 & 3.40 \\
\hline Wheat middlings [CR] & 141 & 0 & 100 & 89.0 & 2.09 & 0.15 & 0.91 & 0.28 & 0.06 & 0.07 & 1.10 \\
\hline Wheat [SID] & 158 & 0 & 100 & 88.0 & 3.17 & 0.05 & 0.41 & 0.12 & 0.06 & 0.07 & 0.78 \\
\hline Wheat, hard, grain [CR] & 158 & 0 & 100 & 88.0 & 3.17 & 0.05 & 0.41 & 0.12 & 0.06 & 0.07 & 0.78 \\
\hline Fat, animal/vegetable & 545 & 1 & 100 & 99.0 & 7.92 & 0.00 & 0.00 & 0.00 & 0.00 & 0.00 & 0.00 \\
\hline Dical. phos. & 426 & 0 & 100 & 100.0 & 0.00 & 22.00 & 18.50 & 18.50 & 0.06 & 0.01 & 0.00 \\
\hline Limestone & 52 & 0 & 100 & 100.0 & 0.00 & 38.00 & 0.00 & 0.00 & 0.05 & 0.03 & 0.00 \\
\hline DI-Methionine & 2633 & 0 & 100 & 99.7 & 5.02 & 0.00 & 0.00 & 0.00 & 0.00 & 0.00 & 0.00 \\
\hline L-Lysine HCl & 1399 & 0 & 100 & 98.5 & 4.12 & 0.00 & 0.00 & 0.00 & 0.00 & 0.00 & 0.00 \\
\hline L-Threonine & 1754 & 0 & 100 & 99.5 & 3.49 & 0.00 & 0.00 & 0.00 & 0.00 & 0.00 & 0.00 \\
\hline L-Tryptophan & 16300 & 0 & 0 & 99.0 & 5.71 & 0.00 & 0.00 & 0.00 & 0.00 & 0.00 & 0.00 \\
\hline L-Valine & 7000 & 0 & 0 & 99.7 & 5.26 & 0.00 & 0.00 & 0.00 & 0.00 & 0.00 & 0.00 \\
\hline Common salt & 117 & 0.4 & 0.4 & 100.0 & 0.00 & 0.30 & 0.00 & 0.00 & 39.00 & 60.00 & 0.00 \\
\hline Vitamin premix & 3600 & 0.25 & 0.25 & 100.0 & 0.00 & 0.00 & 0.00 & 0.00 & 0.00 & 0.00 & 88.00 \\
\hline Mineral premix & 1060 & 0.09 & 0.09 & 100.0 & 0.00 & 0.00 & 0.00 & 0.00 & 0.00 & 0.00 & 0.00 \\
\hline
\end{tabular}

Table 2. Ingredient protein and amino acid compositions (\%). Feeds for growing broilers were formulated with standardized illeal digestibility (SID) values and for mature birds with values from cecectomized roosters (CR)

\begin{tabular}{|c|c|c|c|c|c|c|c|c|c|}
\hline Ingredients & $\begin{array}{l}\text { Crude } \\
\text { Protein }\end{array}$ & $\begin{array}{l}\text { True } \\
\text { Protein }\end{array}$ & dLYS & dMET & dTSAA & dTHR & dTRP & dARG & dVAL \\
\hline Corn [SID] & 7.50 & 6.82 & 0.22 & 0.17 & 0.32 & 0.25 & 0.06 & 0.37 & 0.39 \\
\hline Corn [CR] & 7.50 & 6.82 & 0.20 & 0.17 & 0.32 & 0.24 & 0.06 & 0.36 & 0.37 \\
\hline Soybean meal (48\% CP) [SID] & 47.80 & 43.13 & 2.72 & 0.64 & 1.21 & 1.70 & 0.62 & 3.35 & 2.38 \\
\hline Soybean meal $(48 \% \mathrm{CP})[\mathrm{CR}]$ & 47.80 & 43.13 & 2.74 & 0.65 & 1.24 & 1.77 & 0.62 & 3.34 & 2.45 \\
\hline Corn DDGS [SID] & 25.90 & 24.80 & 0.48 & 0.43 & 0.77 & 0.70 & 0.17 & 0.95 & 1.04 \\
\hline Corn DDGS [CR] & 27.00 & 24.80 & 0.56 & 0.44 & 0.91 & 0.66 & 0.15 & 0.87 & 1.06 \\
\hline Wheat middlings [CR] & 15.00 & 13.49 & 0.56 & 0.10 & 0.25 & 0.39 & 0.17 & 0.87 & 0.64 \\
\hline Wheat [SID] & 13.50 & 11.86 & 0.34 & 0.23 & 0.50 & 0.30 & 0.15 & 0.51 & 0.62 \\
\hline Wheat, hard, grain [CR] & 13.50 & 11.86 & 0.33 & 0.22 & 0.48 & 0.29 & 0.16 & 0.52 & 0.60 \\
\hline Fat, animal/vegetable & 0.00 & 0.00 & 0.00 & 0.00 & 0.00 & 0.00 & 0.00 & 0.00 & 0.00 \\
\hline Dical. phos. & 0.00 & 0.00 & 0.00 & 0.00 & 0.00 & 0.00 & 0.00 & 0.00 & 0.00 \\
\hline Limestone & 0.00 & 0.00 & 0.00 & 0.00 & 0.00 & 0.00 & 0.00 & 0.00 & 0.00 \\
\hline DI-Methionine & 65.80 & 97.00 & 0.00 & 99.00 & 99.00 & 0.00 & 0.00 & 0.00 & 0.00 \\
\hline L-Lysine $\mathrm{HCl}$ & 134.76 & 78.00 & 78.00 & 0.00 & 0.00 & 0.00 & 0.00 & 0.00 & 0.00 \\
\hline L-Threonine & 85.81 & 97.00 & 0.00 & 0.00 & 0.00 & 98.50 & 0.00 & 0.00 & 0.00 \\
\hline L-Tryptophan & 92.27 & 97.00 & 0.00 & 0.00 & 0.00 & 0.00 & 98.00 & 0.00 & 0.00 \\
\hline L-Valine & 87.55 & 97.00 & 0.00 & 0.00 & 0.00 & 0.00 & 0.00 & 0.00 & 96.50 \\
\hline Common salt & 0.00 & 0.00 & 0.00 & 0.00 & 0.00 & 0.00 & 0.00 & 0.00 & 0.00 \\
\hline Vitamin premix & 0.00 & 0.00 & 0.00 & 0.00 & 0.00 & 0.00 & 0.00 & 0.00 & 0.00 \\
\hline Mineral premix & 0.00 & 0.00 & 0.00 & 0.00 & 0.00 & 0.00 & 0.00 & 0.00 & 0.00 \\
\hline
\end{tabular}


Comparison of formulation costs for poultry feeds based on crude protein versus the digestible lysine to protein ratio Broch J. et al.

Table 3. Nutritional restrictions based on breeder management guide recommendations used in the formulations.

\begin{tabular}{|c|c|c|c|c|c|c|c|c|c|c|c|c|}
\hline \multirow[t]{3}{*}{ Ingredients } & \multirow{2}{*}{\multicolumn{2}{|c|}{$\begin{array}{c}\text { Ross } 308 \\
\text { Broiler } \\
\begin{array}{c}\text { Starter } \\
1-10 \mathrm{~d}\end{array} \\
\end{array}$}} & \multirow{2}{*}{\multicolumn{2}{|c|}{$\begin{array}{c}\text { Cobb } 700 \\
\text { Broiler }\end{array}$}} & \multirow{2}{*}{\multicolumn{2}{|c|}{$\begin{array}{c}\begin{array}{c}\text { HY-LINE } \\
\text { W-36 }\end{array} \\
\text { Starter } 1 \\
\text { (0-3 wks) }\end{array}$}} & \multirow{2}{*}{\multicolumn{2}{|c|}{$\begin{array}{l}\text { ROSS 708- } \\
\begin{array}{c}\text { Female } \\
\text { Parent }\end{array} \\
\text { Breeder } 1\end{array}$}} & \multirow{2}{*}{\multicolumn{2}{|c|}{$\begin{array}{c}\text { HY-LINE } \\
\text { W-36 } \\
\text { Peaking }\end{array}$}} & \multirow{2}{*}{\multicolumn{2}{|c|}{$\begin{array}{c}\begin{array}{c}\text { HY-LINE } \\
\text { W-36 }\end{array} \\
\text { Layer } 5 \\
(<80 \%)\end{array}$}} \\
\hline & & & & & & & & & & & & \\
\hline & Min. & Max. & Min. & Max. & Min. & Max. & Min. & Max. & Min. & Max. & Min. & Max. \\
\hline Met. Energy (Kcal g-1) & 3.00 & 100 & 3.20 & 100 & 2.98 & 100 & 2.80 & 100 & 2.84 & 100 & 2.78 & 100 \\
\hline Crude protein (\%) & 23.00 & 100 & 18.00 & 100 & 20.00 & 100 & 15.00 & 100 & 19.05 & 100 & 15.86 & 100 \\
\hline True protein (\%) & 0.00 & 100 & 0.00 & 100 & 0.00 & 100 & 0.00 & 100 & 0.00 & 100 & 0.00 & 100 \\
\hline $\mathrm{Ca}(\%)$ & 0.96 & 100 & 0.76 & 100 & 1.00 & 100 & 3.00 & 100 & 4.94 & 100 & 5.00 & 100 \\
\hline $\begin{array}{l}\text { Avail. phosphorus } \\
\text { (\%) }\end{array}$ & 0.48 & 100 & 0.38 & 100 & 0.50 & 100 & 0.35 & 100 & 0.58 & 100 & 0.41 & 100 \\
\hline $\mathrm{Na}(\%)$ & 0.16 & 100 & 0.15 & 100 & 0.18 & 100 & 0.15 & 100 & 0.21 & 100 & 0.19 & 100 \\
\hline $\mathrm{Cl}(\%)$ & 0.16 & 100 & 0.15 & 100 & 0.18 & 100 & 0.16 & 100 & 0.21 & 100 & 0.19 & 100 \\
\hline Choline (ug g-1) & 1.70 & 100 & 0.35 & 100 & 0.11 & 100 & 1.20 & 100 & 0.11 & 100 & 0.11 & 100 \\
\hline dLYS (\%) & 1.28 & 100 & 0.93 & 100 & 1.05 & 100 & 0.60 & 100 & 0.96 & 100 & 0.73 & 100 \\
\hline dMET (\%) & 0.51 & 100 & 0.38 & 100 & 0.47 & 100 & 0.35 & 100 & 0.47 & 100 & 0.35 & 100 \\
\hline dTSAA (\%) & 0.95 & 100 & 0.72 & 100 & 0.74 & 100 & 0.56 & 100 & 0.81 & 100 & 0.60 & 100 \\
\hline dTHR (\%) & 0.86 & 100 & 0.63 & 100 & 0.69 & 100 & 0.47 & 100 & 0.67 & 100 & 0.51 & 100 \\
\hline dTRP (\%) & 0.20 & 100 & 0.17 & 100 & 0.18 & 100 & 0.14 & 100 & 0.20 & 100 & 0.15 & 100 \\
\hline dARG (\%) & 1.37 & 100 & 1.00 & 100 & 1.12 & 100 & 0.79 & 100 & 1.00 & 100 & 0.76 & 100 \\
\hline dVAL (\%) & 0.96 & 100 & 0.71 & 100 & 0.76 & 100 & 0.56 & 100 & 0.84 & 100 & 0.64 & 100 \\
\hline
\end{tabular}

\section{Results and discussion}

Using the dLys to TP ratio versus CP minimums for the model diets chosen changed formula costs by $\$+10.96$ (Ross 308 Broiler - Starter), $\$+1.57$ (Cobb 700 Broiler - Finisher), \$-3.95 (Hy-Line W-36- Starter 1), \$-4.26 (Ross 708 - Breeder 1), \$+0.05 (Hy-Line W36 Peaking) and \$-2.82 (Hy-Line W36 - Layer 5) per ton of feed (Tables 4 and 5).

The magnitude of the changes was dependent on the imbalance present in the feeds from using the breeder management guide recommendations. The Broiler Starter was the most imbalanced of the growing phase diets (Table 4) with an insufficient amount of TP, having a TP: dLYS ratio of 16.24:1. To bring the feed to the recommended 17.92:1 cost \$10.96 per ton and caused a change in the formulation from corn-soybean meal based to wheat-soybean meal based. The Breeder 1 was the most imbalanced of the laying phase diets (Table 5) with an excessive amount of TP, having a TP: dLYS ratio of 20.86. To bring the feed closer to the recommended 17.92:1 saved $\$ 4.26$ per ton and caused a change in the formulation of increases in corn and wheat middlings and decreases in soybean meal and wheat. So, potential savings from just feed costs could be up to $\$ 4 /$ ton based on 2017 prices. The least-cost diet provided extra protein. To lower TP further (and reduce N pollution) would increase costs further. This shows the cost of reducing pollution with these particular ingredient prices. Having a dLys: TP $>17.92$ should increase waste while dLys: TP $<17.92$ should reduce performance. 
Comparison of formulation costs for poultry feeds based on crude protein versus the digestible lysine to protein ratio Broch J. et al.

Table 4. Results of formulation with crude protein minimums $(C P=K)$ versus a minimum digestible lysine to true protein ratio (LYS:TP). Formulations for growing birds based on standardized illeal digestibility (SID) values

\begin{tabular}{|c|c|c|c|c|c|c|}
\hline & \multicolumn{2}{|c|}{$\begin{array}{c}\text { Ross } 308 \text { Broiler } \\
\text { Starter } 1-10 \mathrm{~d}\end{array}$} & \multicolumn{2}{|c|}{$\begin{array}{l}\text { Cobb } 700 \text { Broiler } \\
\text { Finisher } 2(43+ \\
\text { d) }\end{array}$} & \multicolumn{2}{|c|}{$\begin{array}{c}\text { HY-LINE W-36 } \\
\text { Starter } 1(0-3 \\
\text { wks) }\end{array}$} \\
\hline & $\mathrm{CP}=\mathrm{K}$ & LYS:TP & $\mathrm{CP}=\mathrm{K}$ & LYS:TP & $\mathrm{CP}=\mathrm{K}$ & LYS:TP \\
\hline Formula cost ( $\$ /$ Ton) & 249.41 & 260.37 & 226.56 & 228.13 & 197.49 & 193.54 \\
\hline Cost difference (\$/Ton) & \multicolumn{2}{|c|}{10.96} & \multicolumn{2}{|c|}{1.57} & \multicolumn{2}{|c|}{-3.95} \\
\hline Feed Ingredient Composition & \multicolumn{2}{|c|}{$(\%)$} & \multicolumn{2}{|c|}{ (\%) } & \multicolumn{2}{|c|}{ (\%) } \\
\hline Corn $[S I D]$ & 33.35 & 0.00 & 39.47 & 25.47 & 8.65 & 42.83 \\
\hline Soybean meal (48\%CP) [SID] & 32.38 & 35.21 & 19.81 & 19.21 & 12.41 & 13.85 \\
\hline Corn DDGS [SID] & 5.00 & 5.00 & 5.00 & 5.00 & 5.00 & 5.00 \\
\hline Wheat [SID] & 22.45 & 51.42 & 27.99 & 42.09 & 56.17 & 21.73 \\
\hline Fat, animal/vegetable & 2.44 & 4.31 & 4.22 & 4.73 & 3.06 & 1.81 \\
\hline Dical. phos. & 1.67 & 1.66 & 1.21 & 1.22 & 1.49 & 1.47 \\
\hline Limestone & 1.25 & 1.20 & 1.08 & 1.07 & 12.11 & 12.15 \\
\hline DI-Methionine & 0.30 & 0.23 & 0.18 & 0.16 & 0.11 & 0.15 \\
\hline L-Lysine $\mathrm{HCl}$ & 0.29 & 0.16 & 0.24 & 0.23 & 0.20 & 0.21 \\
\hline L-Threonine & 0.13 & 0.07 & 0.08 & 0.08 & 0.07 & 0.07 \\
\hline Common salt & 0.40 & 0.40 & 0.40 & 0.40 & 0.40 & 0.40 \\
\hline Vitamin premix & 0.25 & 0.25 & 0.25 & 0.25 & 0.25 & 0.25 \\
\hline Mineral premix & 0.09 & 0.09 & 0.09 & 0.09 & 0.09 & 0.09 \\
\hline \multicolumn{7}{|c|}{ Feed Nutrient Composition } \\
\hline Dry matter (\%) & 88.23 & 89.07 & 88.20 & 88.53 & 90.03 & 89.22 \\
\hline Met. energy ( $\mathrm{kcal} \mathrm{g}^{-1}$ ) & 3.00 & 3.00 & 3.20 & 3.20 & 2.78 & 2.78 \\
\hline Crude protein $(\%)$ & 23.00 & 25.49 & 18.00 & 18.56 & 15.86 & 14.50 \\
\hline True protein (\%) & 20.78 & 22.94 & 16.22 & 16.67 & 14.17 & 13.08 \\
\hline $\mathrm{Ca}(\%)$ & 0.96 & 0.96 & 0.76 & 0.76 & 5.00 & 5.00 \\
\hline Avail. P (\%) & 0.48 & 0.48 & 0.38 & 0.38 & 0.41 & 0.41 \\
\hline Ca:P g g-1 & 0.00 & 0.00 & 0.00 & 0.00 & -2.09 & -2.09 \\
\hline $\mathrm{Na} \%$ & 0.20 & 0.21 & 0.20 & 0.20 & 0.21 & 0.20 \\
\hline $\mathrm{Cl} \%$ & 0.28 & 0.29 & 0.29 & 0.29 & 0.30 & 0.29 \\
\hline Choline ug $\mathrm{g}^{-1}$ & 1.85 & 1.79 & 1.61 & 1.55 & 1.28 & 1.43 \\
\hline dLYS (\%) & 1.28 & 1.28 & 0.93 & 0.93 & 0.73 & 0.73 \\
\hline dMET (\%) & 0.63 & 0.59 & 0.45 & 0.44 & 0.35 & 0.38 \\
\hline dTSAA (\%) & 0.95 & 0.95 & 0.72 & 0.72 & 0.60 & 0.60 \\
\hline dTHR (\%) & 0.86 & 0.86 & 0.63 & 0.63 & 0.51 & 0.51 \\
\hline dTRP (\%) & 0.26 & 0.31 & 0.20 & 0.21 & 0.18 & 0.15 \\
\hline dARG (\%) & 1.37 & 1.49 & 1.00 & 1.00 & 0.78 & 0.78 \\
\hline dVAL (\%) & 1.09 & 1.21 & 0.85 & 0.87 & 0.73 & 0.68 \\
\hline TP:dLYS $g g^{-1}$ & 16.24 & 17.92 & 17.44 & 17.92 & 19.41 & 17.92 \\
\hline
\end{tabular}


Comparison of formulation costs for poultry feeds based on crude protein versus the digestible lysine to protein ratio Broch J. et al.

Table 5. Results of formulation with crude protein minimums $(C P=K)$ versus a minimum digestible lysine to true protein ratio (LYS:TP). Formulations for mature birds based on ceacectomized rooster (CR) values

\begin{tabular}{|c|c|c|c|c|c|c|}
\hline & \multicolumn{2}{|c|}{$\begin{array}{l}\text { ROSS } 708 \\
\text { Breeder } 1\end{array}$} & \multicolumn{2}{|c|}{$\begin{array}{l}\text { HY-LINE W36 } \\
\text { Peaking }\end{array}$} & \multicolumn{2}{|c|}{$\begin{array}{l}\text { HY-LINE W-36 } \\
\text { Layer } 5 \text { (>80\%) }\end{array}$} \\
\hline & $\mathrm{CP}=\mathrm{K}$ & LYS:TP & $C P=K$ & LYS:TP & $C P=K$ & LYS:TP \\
\hline Formula cost $\left(\$\right.$ Ton $\left.^{-1}\right)$ & 174.33 & 170.07 & 229.49 & 229.53 & 193.0009 & 190.1747 \\
\hline Cost difference $\left(\$\right.$ Ton $\left.^{-1}\right)$ & \multicolumn{2}{|c|}{-4.26} & \multicolumn{2}{|c|}{0.05} & \multicolumn{2}{|c|}{-2.82} \\
\hline Feed Ingredient Composition & \multicolumn{2}{|c|}{$(\%)$} & \multicolumn{2}{|l|}{$(\%)$} & \multicolumn{2}{|c|}{$(\%)$} \\
\hline Corn [CR] & 0.00 & 2.47 & 3.96 & 3.47 & 12.72 & 38.03 \\
\hline Soybean meal (48\% CP) [CR] & 2.97 & 0.00 & 19.80 & 19.78 & 11.14 & 12.42 \\
\hline Corn DDGS [CR] & 10.00 & 10.00 & 10.00 & 10.00 & 10.00 & 10.00 \\
\hline Wheat middlings [CR] & 8.11 & 10.60 & 0.00 & 0.00 & 0.00 & 0.00 \\
\hline Wheat, hard, grain [CR] & 68.53 & 66.37 & 45.05 & 45.54 & 48.40 & 22.68 \\
\hline Fat, animal/vegetable & 1.00 & 1.00 & 6.11 & 6.12 & 3.00 & 2.12 \\
\hline Dicalcium phosphate & 0.99 & 0.99 & 2.27 & 2.27 & 1.38 & 1.37 \\
\hline Limestone & 7.16 & 7.18 & 11.45 & 11.45 & 12.19 & 12.21 \\
\hline DI-Methionine & 0.13 & 0.15 & 0.25 & 0.24 & 0.11 & 0.13 \\
\hline L-Lysine $\mathrm{HCl}$ & 0.25 & 0.34 & 0.26 & 0.26 & 0.24 & 0.24 \\
\hline L-Threonine & 0.12 & 0.17 & 0.11 & 0.11 & 0.08 & 0.07 \\
\hline Common salt & 0.40 & 0.40 & 0.40 & 0.40 & 0.40 & 0.40 \\
\hline Vitamin premix & 0.25 & 0.25 & 0.25 & 0.25 & 0.25 & 0.25 \\
\hline \multirow[t]{2}{*}{ Mineral premix } & 0.09 & 0.09 & 0.09 & 0.09 & 0.09 & 0.09 \\
\hline & \multicolumn{4}{|c|}{ Feed Nutrient Composition } & & \\
\hline Dry matter (\%) & 89.71 & 89.71 & 90.80 & 90.81 & 90.24 & 89.64 \\
\hline Met. energy ( $\left.\mathrm{kal} \mathrm{g}^{-1}\right)$ & 2.80 & 2.80 & 2.84 & 2.84 & 2.78 & 2.78 \\
\hline Crude protein (\%) & 15.00 & 14.02 & 19.05 & 19.07 & 15.86 & 14.90 \\
\hline True protein (\%) & 13.42 & 12.52 & 17.19 & 17.20 & 14.26 & 13.49 \\
\hline $\mathrm{Ca}(\%)$ & 3.00 & 3.00 & 4.94 & 4.94 & 5.00 & 5.00 \\
\hline Available phosphate (\%) & 0.35 & 0.35 & 0.58 & 0.58 & 0.41 & 0.41 \\
\hline $\mathrm{Ca}: \mathrm{Pg} \mathrm{g}^{-1}$ & -1.15 & -1.15 & -1.89 & -1.89 & -2.09 & -2.09 \\
\hline $\mathrm{Na}(\%)$ & 0.23 & 0.23 & 0.22 & 0.22 & 0.22 & 0.21 \\
\hline $\mathrm{Cl}(\%)$ & 0.31 & 0.31 & 0.30 & 0.30 & 0.30 & 0.29 \\
\hline Choline ug $\mathrm{g}^{-1}$ & 1.27 & 1.22 & 1.52 & 1.52 & 1.39 & 1.51 \\
\hline dLYS (\%) & 0.60 & 0.60 & 0.96 & 0.96 & 0.73 & 0.73 \\
\hline dMET (\%) & 0.35 & 0.35 & 0.52 & 0.52 & 0.35 & 0.36 \\
\hline dTSAA (\%) & 0.61 & 0.59 & 0.81 & 0.81 & 0.61 & 0.60 \\
\hline dTHR (\%) & 0.47 & 0.47 & 0.67 & 0.67 & 0.51 & 0.51 \\
\hline dTRP (\%) & 0.16 & 0.14 & 0.21 & 0.21 & 0.17 & 0.15 \\
\hline dARG (\%) & 0.62 & 0.54 & 1.00 & 1.00 & 0.76 & 0.76 \\
\hline dVAL (\%) & 0.64 & 0.58 & 0.88 & 0.88 & 0.72 & 0.69 \\
\hline TP:dLYS $g^{-1}$ & 22.36 & 20.86 & 17.91 & 17.92 & 19.53 & 18.48 \\
\hline
\end{tabular}


The original imbalances (Table 3) probably came from the way that most requirements have historically been derived from research data. Different researchers have chosen different ways to resolve the cognitive dissonance arising when changes in average growth not different at some arbitrary level of significance are therefore assumed to be equal(12, 13). One approach resulting in inadequate levels of TP could be called the "not significantly different from maximum performance approach".

Payne ${ }^{(14)}$ used this approach to analyze a data set from experiments designed to compare broiler responses to feeds with supplemental amino acids and reduced crude protein levels in. The experiments included were all based on the hypothesis that crude protein levels could be lowered by supplementing synthetic amino acids. Payne (2007) (14) presented bar graphs showing that within experiments there were few detectable differences between lower crude protein diets with higher purified amino acid supplementation and higher crude protein with lower amino acid supplementation. Payne ${ }^{(14)}$ rightly concluded (based on the assumption that not finding significant differences means the treatments were equal): "It seems that the crude protein levels in broiler diets can be reduced by 3 to $4 \%$ percentage points without sacrificing performance provided that free amino acids are supplemented in the diet to equal the amino acid levels in a conventional diet".

Pesti(15) re-evaluated the same data set, but with a different approach. A meta-analyses revealed that there were indeed reductions in performance when crude protein levels were lowered $(p<0.0001)$ when the data were pooled. Crude protein levels resulting in actually sub-maximal, but not significantly different from maximal, performance were detected by a regression approach. It could be demonstrated that feed efficiency was indeed still related to crude protein level in the range of most of the trials studied by Payne(14).

Payne ${ }^{(14)}$ resolved the cognitive dissonance arising between 1) not significantly different indicates the same as equal and 2) not significantly different indicates there may be differences too small to detect by the current experiment, on the side of not significantly different indicates equal. This is an entirely normal way to resolve the dissonance. Pesti ${ }^{(15)}$ on the other hand, demonstrated that there are indeed small differences not detectable by examining individual experiments, also an entirely normal way to resolve the dissonance.

The approach used by Pesti(15) could be called the "margin of safety approach". When diets are formulated on the basis of $\mathrm{CP}$, safety margins are adopted to ensure the supply of AAs often providing a surplus to overcome the problem from differences "not significantly different" in one or even several experiments, to have serious economic implications. The broiler management guides and the tables of nutrition requirements for broilers may suggest high CP levels, these recommendations being in excess of what the average bird really needs. The excess provides the "not significantly different" portion to be sure there is enough.

Nutritionists are well aware of differences in the composition of all the various ingredients that they must use. Most nutritionists specify what might be considered excessive levels of nutrients to account for not only the half of all batches of ingredients 
with below average compositions but for differences in digestibility and environmental conditions including exposure to diseases ${ }^{(7)}$.

The ratio determined by Alhotan and Pesti ${ }^{(7)}$ was from a summary of experiments with growing broiler chickens and is itself an average number with confidence intervals $(4.92 \% \pm 0.51$ for maximum growth or $5.58 \% \pm 0.70$ for minimum feed conversion ratio). This value may be found to be affected by conditions not strictly controlled in the original experiments and so may be subject to future refinements.

It is well known that there are major differences in amino acid requirements within and between poultry species due to several factors (genetics, age, egg production, etc.; Table 3). However, the requirement profiles are similar with only of minor variations (Figure 1). A mature laying hen (Hy-Line W-36 Peaking) and a growing chicken (Cobb 500 Broiler Finisher 2) require almost the same proportions of the essential amino acids as shown in the figure. Therefore, the dLys: TP ratio obtained from growing broiler chicken experiments could be applied to mature hens.

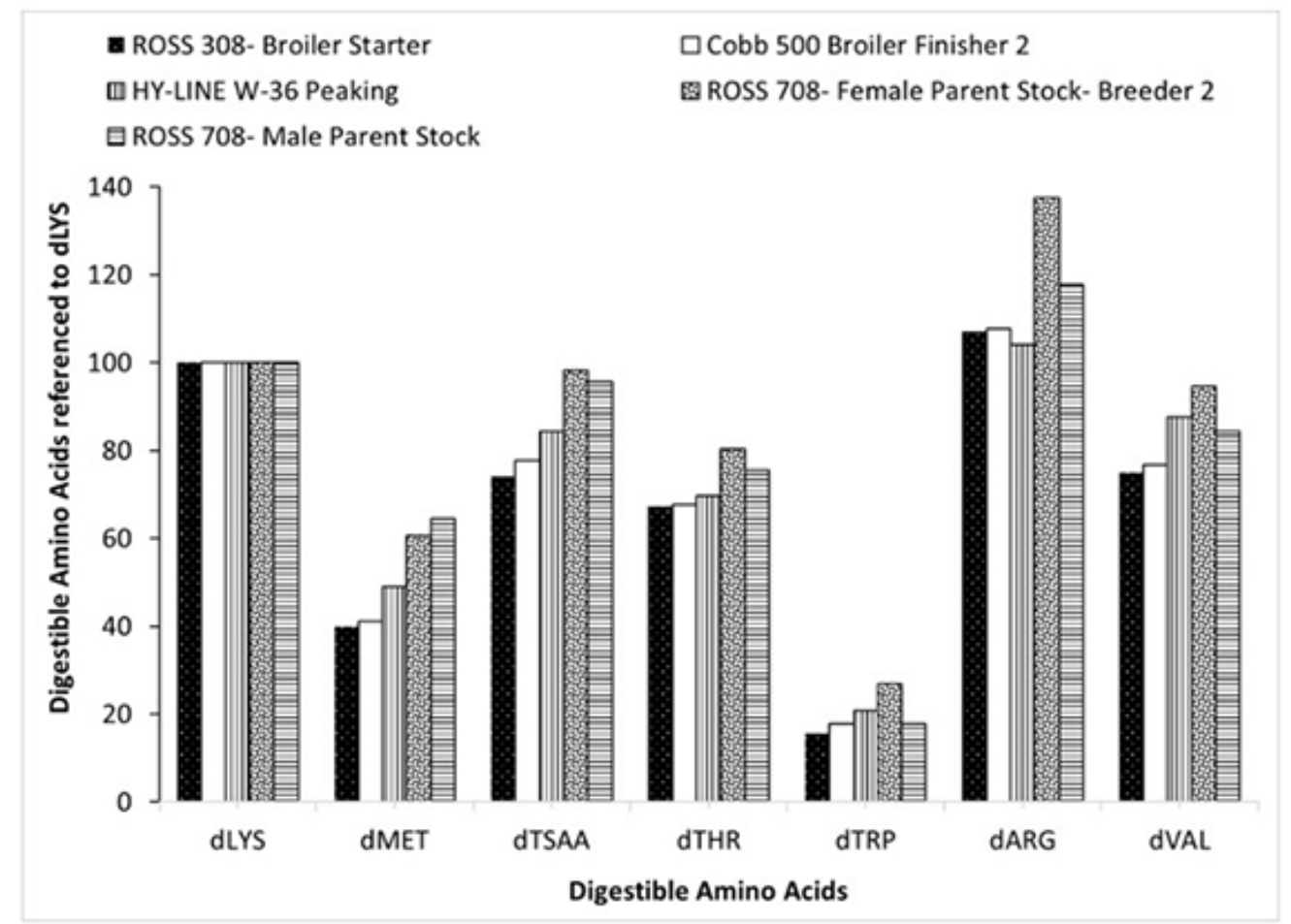

Figure 1. Amino acid profile of the minimum requirements of growing and mature birds [3-5]. Values are on digestible basis and referenced to lysine.

Using TP values is an attempt to account for the birds' non-essential amino acid requirements that are derived from not only the dietary non-essential amino acids, but also from excesses of the essential amino acids. The ratios may change as the birds go from mainly growth to maintenance to egg production. While that remains to be determined, using $5.58 \%$ dLYS of TP for breeders and layers gives an estimate of potential costs and savings using the concept. With the prices from the Spring of 2017 
it appears that such formulation refinements could result in up to $\$ 4 /$ ton savings.

The total impact of better balancing feeds is difficult to estimate since returns could include improvements in performance from not utilizing excess amino acids as energy sources and reduced environmental impact through nitrogenous wastes. Thus, it is important to develop methods that effectively reduce production costs, especially the cost of feeding birds. From the results observed in this work it is suggested that the use of dLys: TP in formulations should receive more attention and be considered as an innovative strategy in the formulation of rations.

\section{Conclusions}

The dLys: TP ratio should be a better method for assuring the birds get adequate EAA and NEAA without being wasteful. Imbalances in recommended crude protein levels may appear to save formulators up to $\sim \$ 4 /$ ton, but the impact of imbalances on performance and environmental contamination is unknown. Potential savings from improved nutritional balance and decreased environmental impacts are hard to measure and will require experiments including magnitude and costs of nitrogenous excretions.

\section{References}

1 Corzo A, Loar RE, Kidd MT. Limitations of dietary isoleucine and valine in broiler chick diets. Poultry Science. 2009;88(9):1934-1938.

2 Vieira SL, Stefanello C, Cemin HS. Lowering the dietary protein levels by the use of synthetic amino acids and the use of a mono component protease. Animal Feed Science and Technology. 2016;(221)262-266.

3 Aviagen Inc. 2007. ROSS 308 Broiler \& Ross 708: Nutrition Specification. Aviagen, Huntsville, AL. http:// en.aviagen.com.

4 Hy-Line International. 2004-2006. Hy-Line W-98 Commercial Management Guide, 2004-2006. Hy-Line Int., West Des Moines, IA.

5 Sriperm N, Pesti GM, Tillman PB. Evaluation of the fixed nitrogen-to-protein (N: P) conversion factor (6.25) versus ingredient specific N: P conversion factors in feedstuffs. Journal Science Food Agriculture. 2011;91(7):1182-1186.

6 Mariotti F, Tome D, Mirand PP. Converting nitrogen into protein: beyond 6.25 and Jones' factors. Food Science Nutrition. 2008;48(2):177-184.

7 Alhotan RA, Pesti GM. Quantitative estimates of the optimal balance between digestible lysine and the true protein contents of broiler feeds. British Poultry Science. 2016;57(4):538-550.

8 Batal A, Dale N. 2015. Feedstuffs. Ingredient analysis tables, University of Georgia, Athens.

9 Evonik Industries. 2005. Optimum ratio of essential amino acids nitrogen to total nitrogen in broiler diets. Facts and Figures, Poultry No. 1586

10 Lysine, H. (1996). Digestibility of essential amino acids for poultry and swine, version 3.3. Heartland 
Comparison of formulation costs for poultry feeds based on crude protein versus the digestible lysine to protein ratio Broch J. et al.

Lysine. Inc., Chicago, IL.

11 Pesti GM, Alhotan R, Vedenov D.; et al. 2016. Windows User-Friendly Feed Formulation 2.0. Can be accessed from the website: https://cfas.ksu.edu.sa/sites/cfas.ksu.edu.sa/files/imce images/ wuffda2.1.xls

12 Greenland S. Null misinterpretation in statistical testing and its impact on health risk assessment. Preventive Medicine. 2011;53(4-5):225-228.

13 Greenland S, Senn SJ, Rothman KJ, Carlin JB, Poole C, Goodman SN, Altman DG. Statistical tests, P values, confidence intervals, and power: a guide to misinterpretations. European Journal Epidemiology. 2016;31(4):337-350.

14 Payne, R.L. The potential for using low crude protein diets for broilers and turkeys. Degussa AminoNews. 2007;6(3) 8:2-13.

15 Pesti GM. Impact of dietary amino acid and crude protein levels in broiler feeds on biological performance. Journal Applied Poultry Research. 2009;18(3):477-486. 